\title{
Ética de lo público: formar para la integridad humana y profesional en el contexto de la educación superior en Colombia*
}

DOI: https://doi.org/10.18046/recs.i31.3251

\begin{abstract}
Public Sector Ethics: Developing Human and Professional Integrity in the Context of Higher Education in Colombia
\end{abstract}

\author{
Edgar A. Vanegas-Carvajal ${ }^{* *}$ \\ Universidad de San Buenaventura (Medellín, Colombia) \\ Verónica Moreno-López ${ }^{* *}$ \\ Universidad de San Buenaventura (Medellín, Colombia) \\ Pablo Echeverri-Rendón ${ }^{* * *}$ \\ Universidad de San Buenaventura (Medellín, Colombia)
}

\begin{abstract}
* Este artículo es el resultado del proyecto de investigación Educar para la integridad humana y profesional. Requerimientos para una adecuada enseñanza de la ética en la educación superior. Análisis desde la USB Medellín, financiado por la Universidad de San Buenaventura, sede Medellín, en el período julio 2017-diciembre 2018. El artículo fue presentado como ponencia en el VI Congreso de Enseñanza de la Ética “Ética de lo público, educación y territorio", organizado por la Red para la Formación Ética y Ciudadana y llevado a cabo los días 6 y 7 de septiembre de 2018 en Cali. Artículo de investigación recibido el 13.11.2018 y aceptado el 29.01.2020.

** Doctor en Filosofía por la Universidad Pontificia Bolivariana (Colombia). Especialista en Pedagogía y Docencia Universitaria y licenciado en Filosofía y Teología de la Universidad de San Buenaventura, sede Bogotá (Colombia). Docente investigador del Centro Interdisciplinario de Estudios Humanísticos, CIDEH, de la Universidad de San Buenaventura, sede Medellín (Colombia). Coinvestigador del proyecto. Grupo de investigación GIDPAD. Correo electrónico: edgar. vanegas@usbmed.edu.co ORCID: https://orcid.org/oooo-ooo1-6124-3944

*** Magíster en Educación de la Universidad de San Buenaventura, sede Medellín (Colombia). Docente-investigadora del CIDEH de la Universidad de San Buenaventura, sede Medellín (Colombia). Investigadora principal del proyecto. Grupo de investigación GIDPAD. Investigadora junior según clasificación Colciencias. Correo electrónico: veronica.moreno@ usbmed.edu.co ORCID: https://orcid.org/oooo-0oo2-1860-4684

**** Magíster en Estudios Urbano-Regionales de la Universidad Nacional de Colombia, sede Medellín (Colombia). Especialista en Derecho Urbano de la Universidad Externado de Colombia (Colombia). Arquitecto de la Universidad Nacional de Colombia, sede Medellín (Colombia). Docente-investigador del CIDEH de la Universidad de San Buenaventura, sede Medellín (Colombia). Coinvestigador del proyecto. Grupo de investigación GIDPAD. Correo electrónico: pablo.echeverri@usbmed.edu.co ORCID: https://orcid.org/oooo-ooo3-0661-9077
\end{abstract}




\section{Cómo citar/How to cite}

Vanegas-Carvajal, Edgar A.; Moreno-López, Verónica; Echeverri-Rendón, Pablo (2020). Ética de lo público: formar para la integridad humana y profesional en el contexto de la educación superior en Colombia. Revista CS, 31, 297-325. https://doi.org/10.18046/recs.i31.3251 


\section{Resumen}

El propósito del presente artículo es identificar estrategias formativas para una adecuada enseñanza de la ética en la educación superior, con el fin de contribuir a la superación del problema de la corrupción en Colombia. Es decir, se busca responder a la pregunta: ¿cómo formar en integridad humana y profesional en la educación superior, para contribuir a la superación del problema de la corrupción en Colombia? Para ello, se proponen tres escenarios: en el primero, se plantea la corrupción como fenómeno sociocultural y moral de connotaciones estructurales; en el segundo, se trazan referentes teóricos sobre el status quaestionis de las prácticas de enseñanza de la ética en Colombia; y, en el tercero, se proponen algunos requerimientos formativos encaminados a mejorar las prácticas de enseñanza en ética de lo público en la educación superior, con el propósito de formar personas íntegras y transparentes, y disminuir los niveles de corrupción en Colombia.

\section{PALABRAS CLAVE:}

corrupción, ética pública, enseñanza de la ética, integridad humana y profesional

The purpose of this article is to identify training strategies for an adequate teaching of ethics in higher education, in order to help overcoming the problem of corruption in Colombia. In other words, it seeks to answer the question: how to develop human and professional academic integrity in higher education, in order to help overcoming the problem of corruption in Colombia? To this end, three scenarios are proposed: 1 ) corruption is considered a socio-cultural and moral phenomenon with structural connotations; 2) theoretical references are drawn about the status quaestionis of ethics teaching practices in Colombia;3) some training requirements are proposed with the aim of improving public ethics teaching practices in higher education and thus, training integral and transparent people and reducing the levels of corruption in Colombia.

\section{KEYWORDS:}

Corruption, Public Sector Ethics, Teaching Ethics, Human and Professional Integrity 



\section{Introducción: \\ fundamentos éticos de la democracia}

Cuando hacemos un análisis de coyuntura a escala regional, nacional o internacional, nos encontramos con que uno de los problemas fundamentales del mundo contemporáneo es el ético. Al hablar de ética no se hace referencia solo al desfalco de las arcas públicas, al engaño y a la corrupción en sus múltiples manifestaciones, sino también al menosprecio del otro; a su objetivación, explotación, cosificación e instrumentalización; a las diferentes formas de discriminación; al racismo; a la injusticia social; al atropello a la dignidad humana y a los derechos fundamentales. En consecuencia, los problemas éticos son muchos más de los que ordinariamente pensamos.

Uno de los fenómenos más acuciantes que amenaza la legitimidad de las democracias de comienzos del siglo XXI es el problema de la corrupción, un fenómeno global que pone en riesgo no solo la estabilidad social y la sana convivencia, sino también el proyecto de nación. Según Transparency International (2019) y los informes preliminares de 2019, Colombia va en descenso en la percepción que se tiene de la corrupción, lo que indica que este es un problema grave en el país. Escándalos de corrupción en los que se encuentran involucrados altos funcionarios públicos, tales como los carteles de la hemofilia, el carrusel de la contratación, el caso Odebrecht, los carteles del azúcar, el cemento, el papel higiénico, la alimentación escolar y los cuadernos, por mencionar solo los que trascienden a los mass media y que son apenas la punta del iceberg del descenso moral en el que se encuentra la sociedad colombiana.

Para enfrentar esta desviación social, la universidad tiene mucho que decir y que ofrecer como institución formadora. La educación humanística en general y la ética pública en particular deben contribuir al desarrollo moral del individuo, de tal modo que le permitan ponderar juicios de valor con base en principios axiológicos, que sirvan "a fines y propósitos que trascienden los intereses personales" (Marreiros; Balça; Acevedo, 2010: 61), como base del compromiso ciudadano y la defensa del bien común.

En efecto, la hipótesis que se quiere sostener en este artículo es que una adecuada enseñanza ética en la educación superior puede ayudar a disminuir los niveles de corrupción en Colombia, al propender por una ética pública a partir de un esquema basado en principios axiológicos como la dignidad humana, el bien común, el respeto por lo público, la integridad, la transparencia, la confianza, la honestidad y la solidaridad. Si bien una adecuada formación en ética pública no es, per se, el antídoto contra la corrupción, formar en ética pública en la educación superior en Colombia puede contrarrestar una de las desviaciones sociales más dañinas en las democracias modernas, y concretamente en la colombiana, como lo es la corrupción. 


\section{Metodología}

Con el fin de responder a la pregunta ¿cómo enfocar la enseñanza de la ética para la formación en integridad académica y profesional en la educación superior a fin de contribuir a la superación del problema de la corrupción en Colombia?, se realizó una revisión crítica de bibliografía, a partir de las categorías de análisis, para proceder al levantamiento de información mediante fuentes documentales y haciendo uso de la técnica de análisis categorial ofrecida por Strauss y Corbin (1998), que propone un procedimiento analítico categorial seguido de un análisis comparativo con muestreo teórico. En este sentido, se privilegia un tipo de estudio no experimental de corte documental-descriptivo, dado que la información recolectada no se interpreta de manera dogmática, sino que se describe después de un análisis crítico.

Para el análisis categorial, se identificaron documentos técnicos, científicos y académicos en bases de datos como Scopus, Science Direct, Dialnet, entre otros. Asimismo, se identificaron informes especializados de entidades y redes de carácter nacional e internacional como Transparency International y Transparency International-Colombia, The Global-Anticorruption Coalition, Organization for Economic Cooperation and Development, Red para la Formación Ética y Ciudadana, Red Nacional de Profesores de Ética, Fundación Étnor y el VI Congreso de Enseñanza de la Ética "Ética de lo público, educación y territorio". La búsqueda de fuentes bibliográficas abarcó un período de veinte años (1998-2018). En total, se revisaron 189 textos, de los cuales se tomaron 51 como referentes. Igualmente, se identificaron otros informes académicos pertinentes.

\section{Resultado y discusión}

\section{La corrupción como enfermedad moral}

Aunque todos los países del mundo tienen problemas de corrupción, cada uno la conceptualiza y caracteriza de modo distinto. Por eso, la tendencia actual apunta a precisar mejor el concepto de corrupción con el fin de lograr una mayor comprensión del fenómeno y poderlo diagnosticar, delimitar y comprender. A partir de una amplia revisión de la literatura sobre la categoría corrupción, se puede decir que el acto corrupto conlleva, per se, por lo menos dos elementos constitutivos: 1) abuso o desviación del poder que se ha encomendado y 2) beneficio o ganancia personal o a terceros. En este sentido y de manera general, Transparency International (2019) 
define la corrupción como 'the abuse of entrusted power for private gain'1. Asimismo, Transparencia por Colombia (2010) la define como 'el abuso del poder delegado para beneficio propio en detrimento de intereses particulares'.

Según Transparency International (2019) y Trasparencia por Colombia, para el año 2017, Colombia ocupó el puesto 96 entre 180 países donde se hizo el estudio. Alcanzó una puntuación de 37/10o, que lo clasificó dentro del grupo de deficiente al lado de Brasil, Panamá y Perú. Estas mismas organizaciones mostraron que, para 2018, Colombia descendió un punto en $2018^{2}$ y pasó del puesto 96 al 99, lo que indica que este es un problema grave en la realidad del país.

En este mismo sentido, Henao e Isaza (2018: 15) conceptualizan la corrupción como "un mal endémico que se incrustó en la sociedad colombiana y se tornó sistémico e inherente a nuestro actuar social. Es un flagelo grotesco, antiestético, despreciable, antidemocrático, tristemente usual, es una enfermedad de nuestra cultura que se debe superar". Este mismo estudio de la Universidad Externado de Colombia concluye que:

Entre 1991 y 2011 este problema costó 189 billones de pesos, unos 9 billones al año. En las últimas encuestas, ha sido señalado como uno de los problemas más graves del país, pues afecta el Gobierno, la Rama Judicial, el sector privado y las organizaciones sociales. (El Tiempo, 2018: 1.4)

Otros estudios recientes como el diagnóstico presentado por Newman y Ángel (2017) para el Centro de Investigaciones Económica y Social de Fedesarrollo concluyen que el problema de la corrupción se ha venido agravando. Se presume que, en la actualidad, se pierden anualmente entre 50 y 55 billones de pesos por este fenómeno, que ha afectado de raíz recursos extraídos de casi todos los sectores sociales: salud, alimentos, infraestructura, educación, vías, etc. Por ello no es exagerado afirmar que la corrupción es un atentado directo a los principios inalienables de la vida humana y la dignidad; dicho de manera directa, la corrupción en cualquiera de sus múltiples manifestaciones es un atentado directo a los derechos fundamentales, al Estado social de derecho y a la democracia misma.

La corrupción es un fenómeno sociocultural de connotaciones estructurales complejas, y en este sentido su comprensión debe tener en cuenta tanto condiciones sociológicas, políticas y culturales -como las planteadas por Thoumi (1999), García

1. El abuso del poder encomendado para beneficio privado (Traducción propia).

2. La escala del índice va de o (muy corrupto) a 100 (muy limpio) (Transparencia por Colombia, 2019). 
y Revelo (2010), Cepeda (2011), la Fiscalía General de la Nación (2018) y el estudio sobre integridad pública en América Latina y el Caribe 2018-2019, realizado por la Organización para la Cooperación y el Desarrollo Económico (OCDE) (2019)-como condiciones antropológicas y organizacionales como las que proponen teóricos como Nye (1967), Sanagustín (2011) y Henao e Isaza (2018).

En efecto, el conocimiento del contexto social, cultural, político e, incluso, geográfico, parece ineludible al momento de diagnosticar y afrontar este flagelo, puesto que la corrupción se modula de forma diferente en cada cultura y sociedad, que bien facilita o favorece ciertas prácticas corruptas sobre otras. Según Newman y Ángel (2017), existen en Colombia unas condiciones sociopolíticas y culturales que benefician distintas prácticas de corrupción. La Figura 1 presenta de forma resumida dichas condiciones.

Con base en lo anterior, la corrupción se puede caracterizar como un fenómeno complejo y multidimensional difícil de aprehender por su naturaleza misma. En consecuencia, su conceptualización, sentido y significación se desplazan de acuerdo con el contexto geográfico, social, cultural, el marco teórico o la forma corrupta

FIGURA 1

Condiciones sociopolíticas e institucionales que facilitan la corrupción en Colombia

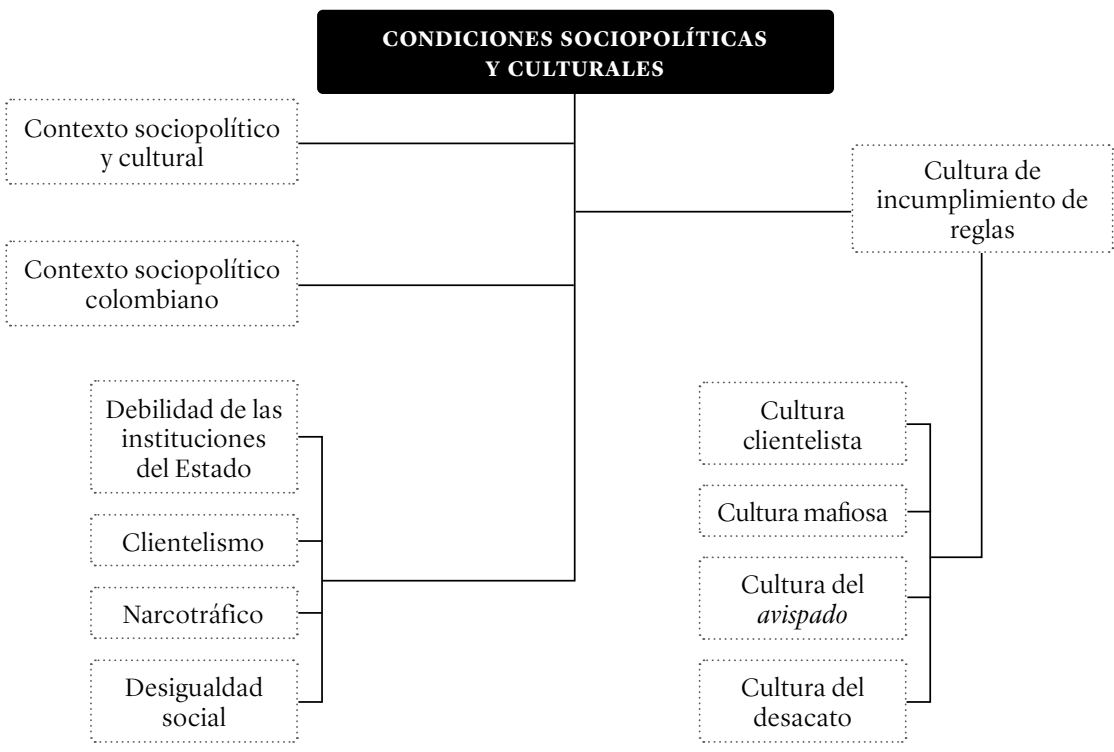


que se quiera resaltar. Para esta indagación y con fines exclusivamente operativos, nos interesa la conceptualización de corrupción ofrecida por Guerrero (2004: 10), cuando afirma que esta "puede ser considerada como una situación de carácter moral", puesto que implica un juicio y una conducta en el orden ético. El mismo autor mantiene esta conceptualización en un artículo sobre corrupción política (CP), publicado de manera conjunta en 2018. En efecto, citando a Álvarez (1997), Laporto y Mauro (1999), Rose-Ackerman (1999) y Peregrini (2011), Guerrero conceptualiza la CP como "un comportamiento que ocurre cuando un funcionario público utiliza el poder discrecional propio de su cargo para su beneficio personal, ocasionando una disminución de los recursos del Estado, y consecuentemente afectando el desarrollo social" (Guerrero; Galván; Granados; García; Vásquez, 2018:546). Decimos entonces que la corrupción es una conducta desviada que pertenece al orden de la moral individual con repercusiones en la moral social.

Estas definiciones están en sintonía con la ofrecida por Transparency International (2009: 14), al entender la corrupción en sentido lato como "el abuso de un poder encomendado para un beneficio personal", y por el Banco Mundial (1993), que conceptualiza la corrupción como "la utilización de un puesto público para obtener beneficios privados". Esta misma definición es adoptada por la Secretaría de Transparencia de la Presidencia de Colombia (2013: 18), al entender la corrupción como "el uso del poder para desviar la gestión de lo público hacia el beneficio privado". En todo caso, sea cual fuere la representación que se tenga de corrupción, esta siempre será una conducta que va en contra de los bienes públicos y que indica un descenso de la ética pública, por donde se va erosionando la moral social y de las organizaciones.

En este punto, parece importante precisar lo que entendemos por ética y ética pública, moral y moral social en los términos planteados en los párrafos anteriores. Primero, hay que advertir al lector que aquí solo se realizará una aproximación a estos conceptos, puesto que, como lo refiere Cortina (2008: 17), "definir términos que tienen una larga historia no es tarea fácil". Hecha esta advertencia, nos podemos arriesgar a decir que la ética en el ámbito formativo es una reflexión constante sobre el actuar, que orienta y evalúa racionalmente las acciones y respuestas ante situaciones concretas de la vida.

Cuando hablamos del concepto griego ethos hacemos referencia a la formación de la conciencia, la forja de carácter y la fijación de hábitos. En palabras de Cortina (2008:17), "la ética es un tipo de saber de los que pretende orientar la acción humana en un sentido racional; es decir, pretende que obremos racionalmente". Dicho en una idea, la ética es una reflexión racional sobre la dimensión moral de la persona. Por su parte, cuando hablamos del término latino mos ('moral'), nos referimos 
igualmente a 'carácter, modo de ser, manera de vivir que configura costumbres'. Desde lo etimológico, podemos decir que ética y moral se utilizan indistintamente como sinónimos, pues ambos conllevan valores, normas y actitudes, pero en la evolución de los términos se han creado matices: con ética se hace referencia al estudio o reflexión sobre el actuar de las personas, es decir, es un estudio racional sobre la moral, mientras que la moral está más relacionada con normas, creencias, costumbres, valores de las personas en una sociedad determinada; así, la moral distingue lo bueno de lo malo.

Ahora bien, ética pública, ética de las organizaciones o ética empresarial-como se suele denominar- hace alusión a un conjunto de principios, valores y deberes aplicados en el ejercicio de las carreras u oficios (deontología). Al decir de Orozco (2012: 197), la ética pública es una "ética aplicada al mundo de las empresas como un saber práctico; es decir, orientada a la acción en diversas dimensiones, económica, social y política. (...) Por tanto, la ética puede caracterizarse como una reflexión, indispensable para el buen gobierno". Como se observa, el ejercicio de la ética pública está orientado a generar confianza a la ciudadanía, partiendo del comportamiento íntegro y transparente de todo profesional (servidor público) en cualquier institución u organización social, sea esta pública, privada o del tercer sector, y que sirve para orientar sus acciones al buen gobierno. Se trata entonces de un obrar responsable, íntegro y transparente de quienes desempeñan funciones públicas, en el marco del mandato constitucional y orientado al bien común.

Por su parte, la categoría moral social o moral pública no deja de ser problemática, como lo plantea Cortina (2008). Esta hace referencia a la conciencia social alcanzada por una determinada sociedad, en el sentido que un grupo social actúa en el marco referencial de un conjunto de principios, valores y convicciones ético-morales al cual llega mediante un consenso, acuerdo o convenio. Así, la moral social o pública es la moral de la mayoría, sin que esto restrinja el derecho al disenso, como la desobediencia civil o la objeción de conciencia. La jurisprudencia constitucional colombiana identifica moral social y moral pública y las conceptualiza como "la que prevalece en cada pueblo en su propia circunstancia" (Corte Constitucional de Colombia, 2017), y que es posible determinar a partir de la Constitución Política.

Hecho este interludio terminológico, se puede afirmar que la corrupción-cualquiera que sea su modalidad o gradualidad-representa un mal moral que socava la respublica, puesto que desconoce el bien común, la dignidad humana y los derechos de los demás. La corrupción es entonces un barómetro que mide la moral no solo personal, sino también social de una determinada población. Dicho de otra manera: la corrupción es la consecuencia final de un descenso de la moral social, o lo que podríamos denominar, parafraseando a Savater (1993), una enfermedad moral que 
denota una pobreza ética en sociedades que se acostumbraron a legitimar prácticas corruptas como si fueran connaturales a la naturaleza humana y a la cultura. Esta enfermedad moral la llaman Bauman y Donskis (2015) adiaforización’3 y Keown (2004) slippery slope (pendiente resbalosa) 4 . En este sentido, se expresan Henao e Isaza (2018:15-16), cuando afirman que la corrupción es:

Un flagelo grotesco, antiestético, despreciable, antidemocrático, tristemente usual, es una enfermedad de nuestra cultura que se debe superar. (...) Las posiciones que la justifican no son más que el reflejo de la pobreza ética de nuestra sociedad, que se acostumbró (...) a no fijar barómetros éticos de altura y de rigor. (...) Ser corrupto supone deficiencias morales e intelectuales que aquejan a quienes toman atajos éticos. (...) Una mente corrupta no es una mente libre, es una mente esclava. Es una mente falseada porque degrada y hace perder la integridad de la persona. (...) La educación es el primer apoyo para superar la crisis ética de la sociedad. (...) Si se mira desde la ética, la corrupción está asociada a prácticas de falsificación.

Este mismo estudio concluyó que la principal modalidad de corrupción en Colombia es el soborno: el $91 \%$ de los encuestados de empresas privadas aceptó haber incurrido en esta práctica, ya sea ofreciendo o recibiendo dinero, regalo o dádiva a cambio de un favor que se necesitaba; el promedio de soborno es del $17 \%$. El argumento que se ofreció para esta práctica fue que era algo propio de la cultura, ya que formaba parte del sistema. Este estudio coincidió con el de Newman y Ángel (2017: 18), cuando afirman que "entre los delitos que se relacionan con formas de corrupción [en el período estudiado 2009-2016], el más frecuente corresponde a la forma de corrupción de soborno (por el delito de cohecho por dar u ofrecer), seguido de apropiación de bienes públicos o bienes de uso privativo y extorsión". Pero también otros tipos de corrupción con sanción penal o disciplinar son muy comunes en Colombia, como "la extorsión, la malversación de fondos, el fraude, el tráfico de influencias, los conflictos de intereses, el nepotismo, la colusión privada y el uso de información privada para tomar decisiones públicas o privadas" (Newman; Ángel, 2017: 17).

Pero ¿qué es lo que genera la corrupción en Colombia? La investigación de Henao e Isaza (2018) plantea principalmente tres factores: primero, condiciones sociopolíticas y culturales (pérdida de los fundamentos éticos de la democracia,

3. Neologismo muy utilizado por Zygmunt Bauman al referirse a las sociedades líquidas, que consiste en 'declarar ciertas acciones o ciertos objetos de una acción en moralmente neutros o irrelevantes, exentos de juicio moral' (Bauman; Donskis, 2015: 23).

4. Expresión extrapolada del campo de la bioética "para referirse a una cadena de acontecimientos que concluirían en algo indeseado” (Keown, 2004: 2O). 
clientelismo y narcotráfico); segundo, condiciones institucionales (en materia de contratación, debilidad de las instituciones, falta de mecanismos de rendición de cuentas); y tercero, cultura de incumplimiento de reglas (falta de formación moral y cultura ciudadana y falta de sanción social, cultura del más vivo o del atajo). En este sentido, la corrupción no solo es "una enfermedad de nuestra cultura (...) y el reflejo de la pobreza ética de nuestra sociedad, que se acostumbró a no rechazar un fenómeno tan despreciable (...) por no haber sabido fijar el baremo ético de altura y rigor" (Henao; Isaza, 2018: 15), sino que es también el resultado de las profundas desigualdades e injusticias sociales que vivimos los colombianos.

\section{La pregunta por la enseñanza de la ética: del qué al cómo}

Frente al panorama planteado, aparece una pregunta ineludible: ¿por qué el Estado es tan proclive a la corrupción?, Más aún, ¿cómo superar el problema de la corrupción en Colombia? Para dar respuesta a estos planteamientos, han aparecido diversas estrategias que se pueden consolidar en la Figura 2:

FIGURA 2 Alternativas para superar el problema de la corrupción en Colombia

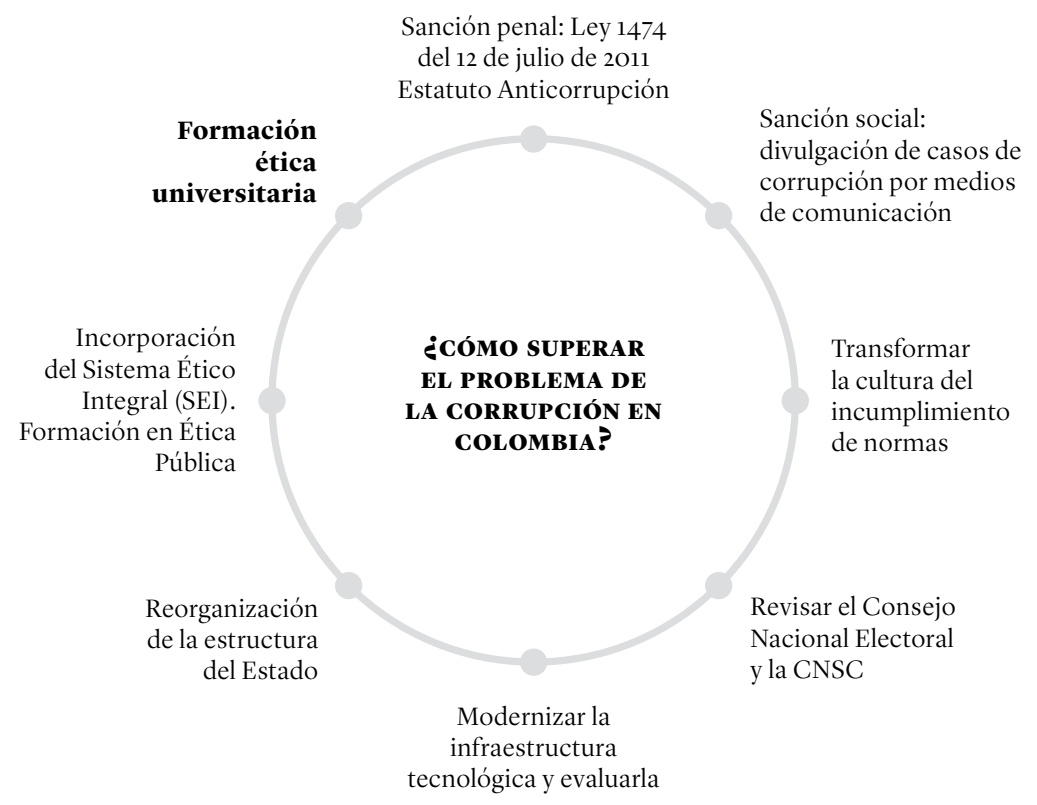


Frente a un problema sistémico y tan generalizado a escala nacional como lo es la corrupción, las iniciativas de lucha deben ser igualmente sistémicas, profundas e integrales. Se cree que soluciones en una sola vía, por sí solas, no son el antídoto; se requiere también transformación social, justicia y equidad, educación ciudadana y cultura por lo público. En este sentido, se pronunció Adela Cortina, cuando Martha Ortiz y Jorge Velásquez (2018: 4) del periódico El Colombiano le preguntaron cómo afrontar el problema de la corrupción en Colombia: "Es un fenómeno muy complejo, pero creo que las dos medidas que se han dicho [regulación y educación] tienen que ir a la vez. (...) Las leyes de transparencia son fundamentales. Esto tiene que venir acompañado de la educación”.

En este mismo sentido, se expresaron Jiménez y Sánchez (2018:2) en una entrevista que les hizo el periódico El Colombiano, de Medellín: "La consulta anticorrupción no es el antídoto contra la corrupción, porque el fenómeno es de tal envergadura que implica un trabajo profundo desde la transformación de la cultura política hacia la legalidad y el respeto por las instituciones”. Más adelante, en esta misma columna, los autores argumentaron que:

El problema no es de normas sino de cultura ciudadana, pues existen 19 leyes que atacan la corrupción, entre ellas 4 aprobatorias de tratados internacionales, y 2 anticorrupción: ley 1990 de 1995 y ley 1474 de 2011, más un Conpes [167 de 2013, Política integral contra la corrupción] y cinco organismos de control y fiscalización.

Sin embargo, en lo que sí coincide la gran mayoría de expertos, instituciones y empresas es en afirmar que la implementación de un Sistema Ético Integral (SEI), unido a una adecuada formación en ética de lo público, constituye una condición de posibilidad ineludible en la lucha contra la corrupción en el caso colombiano. Es decir, que una adecuada formación universitaria en integridad académica y profesional podría ayudar a crear egresados y, por consiguiente, profesionales más íntegros y transparentes para las organizaciones y para la sociedad. Henao e Isaza (2018: 15) plantean que "una educación que se respete tiene que inculcar rectitud a la juventud, decoro, creencia en la perfectibilidad humana y optimismo". También Aranguren (1998: 48) apuesta para que la ética y la moral se sigan enseñando en el ámbito universitario puesto que "el hombre tiene que hacer ese ajustamiento, tiene que iustum facere, es decir, tiene que justificar sus actos, puesto que la justificación es la estructura interna del acto humano".

La pregunta no es entonces si la ética se puede enseñar-aprender o si la enseñanza de la ética compete a la universidad. El problema se desplaza alrededor de qué ética (concepción y enfoques), qué enseñar (contenidos) y cómo enseñar (didácticas) ética 
en la universidad colombiana del siglo XXI, preguntas que implican, por lo demás, cuestionamientos más concretos y que han sido abordadas en nuestro contexto y de manera amplia por la Universidad EAFIT: ¿cuáles son las condiciones pedagógicas de la enseñanza de la ética?, ¿cómo enfocar la enseñanza de la ética para la formación en integridad académica y profesional en el contexto de la educación superior?, ¿se debe enseñar ética o moral?, ¿qué elementos debe contener un currículo orientado a la formación en integridad académica y profesional en la educación superior?, ¿cómo enseñar ética de forma tal que genere un impacto real y positivo en la conducta de los individuos?, ¿la ética que se enseña en la universidad está contribuyendo a propiciar mejores niveles de transparencia y confianza en las personas y en las organizaciones?, ¿cómo se está enseñando la ética en la universidad para contrarrestar el fenómeno de la corrupción en Colombia? Vale la pena agregar que estos planteamientos están siendo abordados por distintas universidades colombianas, entre las que hay que destacar los trabajos de Franco (2017) Estos interrogantes van a tener un punto alto de análisis a finales del siglo XX y comienzos del XXI, cuando emerge un despertar por investigaciones científicas alrededor de la enseñanza de la ética y sus prácticas pedagógicas, a propósito de las "crisis económicas y escándalos de corrupción” (Guerrero-Useda; Gómez-Paternina, 2013: 123). Diversos trabajos científicos han dado cuenta de esta afirmacións. Sin embargo, estos referentes epistemológicos no se corresponden con las prácticas pedagógicas de la enseñabilidad de la ética en la universidad colombiana. Varios de estos estudios concluyen que, aunque existe consenso sobre la importancia capital de la ética en la formación de jóvenes universitarios, sus contenidos, metodologías y didácticas -sus condiciones de enseñabilidad- están por construirse. Dicho en palabras de Orozco (2009: 163), "el aporte que hace la universidad al desarrollo ético y crítico de los estudiantes está comprometido. (...) El problema está en los medios para cumplir esta tarea". Por su parte, Ibáñez (2012) plantea por lo menos tres obstáculos y resistencias actuales para una adecuada enseñanza ética en la universidad.

En primer lugar, la universidad occidental inmersa en un contexto sociocultural de corte capitalista debe responder a unos estándares de mercado y competitividad que la conducen a ofertar programas académicos que den cuenta de las necesidades de la empresa, con el fin de "captar el mayor número de estudiantes que les asegure, no solo la supervivencia, sino también los recursos pretendidos para desarrollar sus proyectos educativos" (Ibáñez, 2012: 196) y ser visibles en los rankings. Lo anterior conlleva el 
riesgo de que la universidad, por el afán de responder a las demandas de mercado y competitividad, sacrifique la formación humana, ética y en valores. Si esto ocurre, la universidad corre el riesgo de quedar reducida a un mero instrumento al servicio de intereses utilitaristas propios de un capitalismo salvaje de corte mercantilista.

El segundo obstáculo para una adecuada formación ética en la universidad parte de resistencias y objeciones de los propios profesores, que ante la falta de un conocimiento suficiente del estatuto epistemológico de la ética, de su estatus como racionalidad científica y de su utilidad práctica para orientar el comportamiento humano, la interpretan de modo desviado como una disciplina donde predomina el subjetivismo, el relativismo e incluso se asocia exclusivamente con una moral de corte religioso. Dicho en palabras de Weiss (2006) y Sanagustín (2011), la ética ha sido mitificada y, en consecuencia, reclama una desmitificación.

En tercer lugar, y como resultado de los dos obstáculos anteriores, emerge la resistencia por parte de estudiantes universitarios a la formación ética al concebir estos cursos como pérdida de tiempo, cursos relleno o inútiles. Primeramente, porque perciben de muchas maneras y por diferentes medios que el mudo real y el ejercicio profesional tienen otras coordenadas en los que la ética puede tener poca utilidad o incluso ser un obstáculo para alcanzar el éxito personal y profesional; otra consideración es que "muchos estudiantes tienen una visión instrumental de la educación universitaria: 'solo sirve lo que es útil para mejorar mis posibilidades de empleo'” (Ibáñez, 2012: 200).

En síntesis, el problema no está centrado en si la ética se puede enseñar-aprender ni en si su enseñabilidad debe ser competencia de la universidad; está claro que enseñar ética sí, pero ¿qué ética y bajo qué estrategias? Así pues, la cuestión se desplaza hacia el enfoque, los contenidos, los modos y las mediaciones, esto es, las nuevas pedagogías y didácticas que reclama la enseñanza universitaria de la ética en las democracias del siglo XXI.

\section{Requerimientos formativos para una adecuada enseñanza en ética de lo público en la educación superior}

Del razonamiento moral a las emociones morales. Lawrence Kohlberg (1927-1987), uno de los mayores teóricos del desarrollo del razonamiento moral, expuso dilemas morales con el fin de descubrir el estadio evolutivo de los niños y situarlos en un determinado estadio moral. Por ejemplo, en el dilema clásico de Heinz donde un hombre debe decidir si ingresa a una farmacia para robar un medicamento que seguro salvaría la vida de su esposa, quien sufre un tipo especial de cáncer terminal, el grupo de niños/adolescentes tenía que justificar las razones por las cuales se debía tomar una determinada decisión, guiando los argumentos con algunas preguntas. 
Basado en este estudio de dilemas morales, Kohlberg (1992) presentó un modelo invariable y rígido de tres niveles con dos estadios cada uno que, de modo ascendente, mostraba el grado de desarrollo moral del niño/adolescente a partir de habilidades cognitivas. Según Kohlberg (1992), cada etapa se caracterizaba por la capacidad cada vez mayor de eliminar el egoísmo y adoptar la postura del otro; es decir, verse como parte de una sociedad. Por ejemplo, en el nivel 3, estadio 6, que corresponde a la moralidad posconvencional, se asumen principios ético-morales universales más allá del contrato social, que permiten a la persona eliminar la heterofobia, la aporofobia ${ }^{6}$ y el misoneísmo, y actuar bajo los principios de justicia, solidaridad y reconocimiento, respetando siempre los derechos fundamentales sin importar origen, etnia, sexo o nivel socioeconómico. Tanto para Cortina (1995) y Habermas (2000) como para Nussbaum (2014a), las sociedades con democracias liberales se encontrarían en este nivel, donde una de las características esenciales tiene que ver con ponerse en el lugar de cualquier otro, ser capaz de imaginar y narrar la realidad del otro, reconocer al otro como un ser valioso igual a mí o ser capaz de pensar en el bien de la nación como un todo.

Kohlberg (1969), inspirado en los estudios del desarrollo de la conciencia moral de Piaget (1965), sostiene que el juicio moral está íntimamente relacionado con el desarrollo del razonamiento y la reflexión. Dicho de otra manera: el juicio moral es producto de un proceso cognitivo que se inscribe en una tradición de corte racionalista. Pero no por ello ha adolecido de amplias críticas y grandes opositores, sobre todo por quienes, como Haidt (2001), consideran que no existe una secuencia universal, rígida e igual para todos en cada estadio; o como Gilligan y Attanucci (1988), que ven error en los hallazgos porque los dilemas fueron aplicados solo en hombres y no en mujeres; o como Hauser (2006), que cuestiona los criterios que se puedan usar para situar a un niño en una de las fases designadas. En este mismo sentido, Guerrero, Galván, Granados, García y Vásquez (2018:546) citan a varios autores para cuestionar el modelo de desarrollo de razonamiento moral de Kohlberg y afirman:

Rest (1986b) señala que no hay una secuencia rígida sino una serie de esquemas estructurados que son flexibles. Aunque hay una relación entre el desarrollo moral y el comportamiento o acción moral (Narváez; Rest, 1995), esta no es proporcionalmente

6. Es un neologismo introducido por la filósofa española Adela Cortina (2017:14) en el libro Aporofobia, el rechazo al pobre: un desafío para la democracia. En la introducción conceptualiza aporofobia como "rechazo, aversión, temor y desprecio hacia los pobres, hacia el desamparado que, al menos en apariencia, no puede devolver nada bueno a cambio. (...) Existe la xenofobia y el racismo, como existe la misoginia, la cristofobia, la islamofobia o la homofobia. (...) Sin embargo, no es de esas actitudes de rechazo de la que queremos hablar en este libro, sino de esas aversiones que se encuentran en la raíz de muchas de ellas y que va aún más lejos: de la aporofobia". 
directa (You; Bebeau, 2013); el desarrollo moral le permite al individuo discriminar cuál de las posibles acciones es la más ética en situaciones donde hay dos o más valores en conflicto (Kohlberg, 1992), sin embargo, no implica que actúe acorde con la decisión ética. (Narváez; Rest, 1995)

En este sentido, se llega a afirmar que la pregunta moral sobre qué es lo correcto es del tipo complejo, ya que implica no solamente elementos conceptuales de índole cognitiva (como los planteados por Kohlberg), sino también elementos de orden volitivo, emocional y contextual. Estudios empíricos actuales han cuestionado el papel de la sola razón en el juicio moral y han revelado la importancia de las emociones. Es decir, en el juicio moral no intervienen únicamente elementos cognitivos, sino que también están implicados elementos emocionales de la antropología y la psicología experimental7.

La gestión de las emociones como fundamento de la educación moral. El racionalismo exacerbado que se origina con las tesis cartesianas tiene consecuencias nefastas para la modernidad, al dejar de lado la importancia que tienen las emociones en la vida personal y social. Esta tradición filosófica utiliza la sola razón con fines instrumentales, a partir del modelo computacional que desconecta la razón de la voluntad, privilegiando la raison raissonnement y descuidando la importancia que tienen las emociones en la vida del sujeto y en las relaciones intersubjetivas. Ya Pascal (1959) había advertido del riesgo de admitir la sola razón en la aprehensión del conocimiento, puesto que hay realidades que la sobrepasan (la moral, la fe, Dios, los primeros principios). Dice Pascal (1959: 62) que "la última resolución de la razón es reconocer que hay infinidad de cosas que la sobrepujan. Es débil del todo si no llega a conocer eso". En esta misma tradición, se sitúan los estudios de Vanegas (2009), al proponer una antropología integral razón-voluntad como vías complementarias de acceder al conocimiento. También Honneth (1997), en su etapa final de producción intelectual, construye su teoría del reconocimiento como una alternativa al cartesianismo, al incluir emociones como el amor, el honor y la solidaridad como condiciones de posibilidad en la construcción intersubjetiva del sujeto ético-político.

Cuando se abordan estudios antropológicos de las emociones (no solo desde la filosofía racionalista o la psicología experimental), se puede llegar a concluir que la razón, por sí sola, no es suficiente para elaborar un juicio moral, sino que, por el contrario, razón y emoción son un continuum difícil de separar y más bien existe una relación de complementariedad. En este sentido, cuando se habla de la ética como saber práctico, lo que se quiere significar es que corresponde a otro tipo de 
racionalidad que implica las emociones humanas como el amor, la empatía, la alegría, la vergüenza, el asco, el miedo, la tristeza, etc., pero sin desaprobar el juicio racional. Esta hipótesis se inscribe en la línea de Díaz (2010: 30) cuando reseña la obra El gobierno de las emociones, de Camps (2011) y afirma que:

La ética no puede prescindir de la parte afectiva o emotiva del ser humano, porque una de sus tareas, es precisamente, poner en orden, organizar y dotar de sentido los afectos o las emociones. La ética no ignora la sensibilidad ni se empeña en reprimirla, lo que pretende es encausarla en la dirección apropiada. (...) En el encauzamiento de las emociones tiene una parte importante la facultad racional, pero no para eliminar el afecto, sino para darle el sentido que conviene más a la vida, tanto individual como colectiva.

Cuando Camps (2011) habla de encauzamiento de las emociones, se está refiriendo a la importancia de educar las emociones para efectos de la ética, tanto desde la claridad cognitiva de la racionalidad analítica como desde "el campo de lo simbólico, del Mythos, del ritual sagrado" (Reyes, 2017: 32). "El mundo de las emociones debe ser trabajado desde el ser humano integral y complejo" (Reyes, 2017: 60), es decir, desde una antropología integral y no solo desde el racionalismo exacerbado o la psicología experimental. Dicho de otra manera: la enseñanza ética actual debe abordar "la emoción como juicio y su importancia en la formación del juicio moral, además del papel de la educación de las emociones en contraste con el intelectualismo moral" (Bedoya; Rey; Romero, 2015: 103), puesto que los seres humanos nos regimos conductualmente no solo por el razonamiento moral, sino también por la valoración emocional. Por eso, Nussbaum (2014b: 481) aconseja conocer "los componentes no cognitivos (las sensaciones, los estados físicos del cuerpo) de las emociones", como detonantes de acciones/inacciones morales, mientras que Lazarus (1991: 135) concluye que "las emociones y la razón se hallan inextricablemente vinculadas en una lógica inexorable".

Las capacidades éticas. Nussbaum (2006:83) define las capacidades como "aquello que las personas son efectivamente capaces de hacer y ser, según una idea intuitiva de lo que es una vida acorde con la dignidad humana". Dicho de otra manera, las capacidades son oportunidades para actuar que implican derechos básicos, y no solo recursos, que surgen como alternativa a los enfoques económicos de corte utilitarista que priman en los debates actuales sobre la calidad de vida. Mientras que para el modelo de desarrollo basado en el PIB per cápita "se necesita de habilidades

8. Realizar un análisis exhaustivo de las emociones a propósito de la ética y la educación moral desbordaría este trabajo; para este efecto, ver Arbeláez-Castaño (2020), Goleman (1996), Le Breton (1998), Mélich (2010) y Rodríguez-Valls (2015). 
básicas, alfabetización y aritmética (...) y habilidades más avanzadas en ciencias de la computación y tecnología" (Nussbaum, 2015:4), para el paradigma de desarrollo humano, por el contrario:

Lo que importa son las oportunidades, o "capacidades" que cada persona tiene, en áreas claves que van desde la vida, la salud, la integridad física hasta la libertad política, la participación política y la educación. (...) Si una nación quiere promover ese tipo de democracia humana, se debe dedicar a la promoción de oportunidades para la vida, la libertad y la búsqueda de la felicidad. (Nussbaum, 2015:5)

En este segundo paradigma, la pregunta de fondo que deben hacerse las democracias modernas es ¿qué habilidades necesita producir un Estado para construir una democracia humana y decente con ciudadanos autónomos y responsables? El punto de partida de Nussbaum (2005) no es otro que el principio inalienable de la dignidad de cada persona, que esta sea respetada tanto por las leyes como por las instituciones y que se reconozca que todos los ciudadanos tienen derechos que se deben traducir en oportunidades para alcanzar el umbral de sus capacidades.

Ahora bien, ¿qué capacidades éticas son particularmente importantes para una ciudadanía democrática y decente? Nussbaum (2005), en las últimas décadas, ha desarrollado principalmente tres: 1 ) capacidad socrática de autocrítica y pensamiento crítico; 2) capacidad de verse a sí mismo como miembro de una nación y un mundo heterogéneos; y 3) capacidad de imaginar (narrar) la realidad del otro. "Se trata de una concepción que promueve en los estudiantes la adquisición de destrezas y habilidades, tanto sociocognitivas como actitudinales para contrarrestar las desigualdades y formar las virtudes democráticas" (Nussbaum, 2005: 104).

En definitiva, ¿cómo formar en capacidades éticas para las democracias del siglo XXI? Se han propuesto dos modelos: el primero afirma que no es posible una enseñanza de las capacidades éticas, sino que estas se aprenden de manera intuitiva e indirecta. En este sentido, "formar en capacidades éticas supone construir contextos que favorezcan, entre otros, la conformación, la asunción y el control de las mismas" (Universidad Pontificia Bolivariana [UPB], 2012: 12). Este esquema se fundamenta en lo que la persona es y sabe hacer como producto de su aprendizaje personal, los conceptos ya adquiridos, su interacción con el entorno, las experiencias personales y las representaciones que la persona posee. El segundo modelo sostiene que las capacidades éticas se pueden enseñar de modo directo por medio del cultivo de la imaginación, la argumentación y la deliberación, esto es, la formación del sentido autocrítico, el juicio analítico, la ejercitación de la empatía y el gobierno de las emociones. "Se trata de un pensamiento orientado a la comprensión de problemas, la 
evaluación de alternativas y la decisión y resolución de los mismos" (UPB, 2012: 13). Para Nussbaum (2012: 20), el pensamiento crítico es "la capacidad de pensar por sí mismos, poseer una mirada crítica sobre las tradiciones y comprender la importancia de los logros y sufrimientos ajenos sustentados en una racionalidad integral y en la comprensión crítica y reflexiva".

Entonces, formar en capacidades éticas-capacidad para argumentar, capacidad de imaginar la realidad del otro y capacidad de deliberar correctamente- aúna el camino para una cultura pública crítica, la enseñanza crítica de la ética, la inculcación del razonamiento moral y la adecuada gestión de las emociones. Lo que se sugiere aquí es que la educación ética universitaria debe ocuparse de formar en capacidades éticas para educar la integralidad de la persona, el pensamiento crítico, la deliberación moral, la gestión de las emociones, la dignidad humana y los derechos fundamentales. Esta alternativa posibilita crear "unas instituciones decentes frente a las continuas presiones ejercidas por el egoísmo, la codicia y la agresividad ansiosa" (Nussbaum, 2014b: 216).

Una ética en tres dimensiones. Vallaeys (2018), en la conferencia de clausura del VI Congreso de Enseñanza de la Ética "Ética de lo público, educación y territorio", presentó una ponencia titulada Ética, responsabilidad e innovación, en la que hizo una crítica a la ética al afirmar: "La ética debe dejar de ser un vano discurso que se lamenta constantemente, pero sin afectar al sistema que critica y abonando finalmente el terreno del conservadurismo reaccionario y potencialmente autoritario". Esta es una ética en primer y único plano que generalmente es dogmática, relativista, individualista e insuficiente para resolver los problemas complejos de coyuntura.

Frente a esta concepción tradicionalista de la enseñanza ética, Vallaeys (2009) concluyó que la ética que requieren las sociedades modernas no puede ser ni una ética de la pulcritud, que conduce a una rigidez socioeducativa y condena la más mínima desviación del comportamiento, ni una ética de la dejadez, que tolera lo intolerable y conduce a una dejadez socioeducativa y hasta adiafórica. Para superar esta ética trasnochada y obsoleta, Vallaeys (2009) propone la idea de una ética en tres dimensiones, a la que comúnmente denomina ética en $3 \mathrm{D}$, esto es, una ética que trascienda el primer plano y que, por el contrario, implique las tres dimensiones humanas: la personal, la pública y la global, cada una dirigida por un principio rector: virtud, justicia y sostenibilidad. Bajo esta tridimensionalidad, la ética es (co)responsable, comunitaria, interpersonal, transparente, íntegra, innovadora y supera el deontologismo de los códigos éticos. La propuesta de Vallaeys se puede esquematizar tal como se muestra en el Cuadro 1: 
CUADRO $1 \mid$ Ética en 3 D

\begin{tabular}{|c|c|c|c|}
\hline & $\begin{array}{l}\text { Primera dimensión } \\
\text { Ética personal }\end{array}$ & $\begin{array}{l}\text { Segunda dimensión } \\
\text { Ética pública }\end{array}$ & $\begin{array}{c}\text { Tercera dimensión } \\
\text { Ética global }\end{array}$ \\
\hline Principio rector & Virtud & Justicia & Sostenibilidad \\
\hline $\begin{array}{l}\text { Valor } \\
\text { predominante }\end{array}$ & Bondad & Mutualidad & Armonía \\
\hline Sujeto del deber & $\begin{array}{c}\text { Autoética } \\
\text { Yo mismo, pensando } \\
\text { en mi actitud frente a } \\
\text { todos los demás }\end{array}$ & $\begin{array}{c}\text { Socioeducativa } \\
\text { Nosotros reunidos, } \\
\text { pensando en todos } \\
\text { nosotros en la comunidad } \\
\text { de derechos }\end{array}$ & $\begin{array}{c}\text { Antropoética } \\
\text { Nosotros reunidos, } \\
\text { pensando en todo } \\
\text { el planeta para las } \\
\text { generaciones futuras }\end{array}$ \\
\hline Lógica normativa & Singularidad & Pluralidad & Totalidad \\
\hline $\begin{array}{l}\text { Ámbito de } \\
\text { legislación }\end{array}$ & $\begin{array}{l}\text { Persona individual } \\
\text { intrapersonal }\end{array}$ & $\begin{array}{l}\text { Sociedad humana } \\
\text { interpresonal }\end{array}$ & $\begin{array}{l}\text { Especie humana } \\
\text { transpersonal }\end{array}$ \\
\hline $\begin{array}{l}\text { Resultado } \\
\text { del deber }\end{array}$ & Acto bueno & Ley justa & Mundo sostenible \\
\hline Foco de atención & Acción & Interacción & Impacto \\
\hline $\begin{array}{l}\text { Responsabilidad } \\
\text { asociada }\end{array}$ & $\begin{array}{c}\text { Responsabilidad } \\
\text { moral }\end{array}$ & Responsabilidad jurídica & Responsabilidad social \\
\hline
\end{tabular}

Fuente: elaboración propia con información tomada de Vallaeys (2009).

\section{Conclusiones 9}

Formar para la integridad humana y profesional en la universidad colombiana implica educar en ética pública para el respeto de la respublica. Educar para lo público implica educar en la generación de confianza, la defensa de la persona, la dignidad humana y los derechos fundamentales, puesto que, en último término, la corrupción, cualquiera que sea su tipología y gradación, constituye un atentado grave a la dignidad humana y a los derechos fundamentales. Gaviria (2012) sostiene que las democracias contemporáneas deben ser evaluables desde un punto ético; es decir, se deben fundamentar en una ética pública sobre una concepción moral de la persona.

9. Estas conclusiones incluyen algunas recomendaciones discutidas y debatidas ampliamente en la mesa "Formación ciudadana, ética de lo público y corrupción" delVICongreso Nacional de Enseñanza de la Ética "Ética de lo público, educación y territorio". En el evento se presentaron algunos avances de este artículo. 
Colombia tiene una larga tradición de violencia, menosprecioy desvalorización de formas de vida distintas a la propia a causa de las profundas injusticias y desigualdades sociales. En un Estado tan injusto y desigual es muy difícil que puedan existir vínculos cívicos auténticos, ya que todas las formas de corrupción son una manifestación de la carencia de vínculos sociales, que se manifiestan en distintas formas de exclusión, intolerancia y menosprecio al otro. Ello impide construir una cultura de respeto por la persona y por los bienes públicos. De aquí extraemos una primera conclusión: el problema de fondo no es la corrupción per se en tanto las estructuras de injusticia y desigualdad en que vivimos conllevan una fractura del lazo social y consecuentemente la vulneración de la dignidad humana y los derechos fundamentales.

Una segunda conclusión que emerge de este estudio es que, si bien la ética por sí misma no constituye un antídoto contra la corrupción, sí representa una estrategia de primer orden contra este flagelo y, por tanto, la enseñanza de una ética pública en el nivel universitario, unida a la implementación de un SEI en las instituciones del Estado, constituye una estrategia pedagógica para la formación de profesionales integros y transparentes para la sociedad. Consecuentemente con lo anterior, la pregunta no es entonces si se enseña ética pública en la universidad, sino que se desplaza alrededor de los requerimientos formativos para una adecuada formación ética.

La pregunta por la enseñanza de la ética es del tipo complejo, puesto que implica no solo elementos conceptuales de orden cognitivo (como los planteados por Kohlberg), sino también de orden emocional y experiencial. Estudios empíricos actuales han cuestionado el papel de la sola razón en la elaboración del juicio moral y han revelado la importancia que tienen las emociones. Esto implica el abordaje de la ética no desde la antropología cultural o la psicología experimental, sino como un continuum que tenga como fundamento la concepción del hombre como un ser multidimensional y complejo; es decir, desde una antropología integral, que tenga en cuenta la dimensión racional y la dimensión emocional de la persona.

Aquí emerge una tercera conclusión que tiene que ver con los requerimientos formativos para una adecuada formación ética en la universidad, los cuales deben ser en varias direcciones -lo que Morin (2004) denomina pensamiento complejo- y también multidimensionales e interdisciplinarios. Víctor Espinosa (2015), director de la Maestría en Ética y Problemas Morales Contemporáneos de la Universidad Minuto de Dios, en Bogotá, afirma que la pedagogía para una adecuada enseñanza en ética pública debe ser resignificada y orientada al aprendizaje contextual y a la resolución de problemas reales de contexto. El presente trabajo ha delineado cuatro enfoques pedagógicos que, sin ser exhaustivos, podría contribuir a formar egresados más íntegros, transparentes y responsables con el erario: el razonamiento moral, las emociones morales, las capacidades humanas y una ética en tres dimensiones. Se 
concluye que estos enfoques permiten un abordaje más completo en la formación para la integridad humana y profesional y determinan un elevado compromiso con principios axiológicos como trabajo decente, transparencia, responsabilidad y confianza, aplicables tanto a los servidores públicos como a los ciudadanos en general. Es decir, un cambio de paradigma en las prácticas pedagógicas de la enseñanza de una ética para lo público en la educación universitaria constituye un aporte significativo en la lucha contra la corrupción y la defensa de lo público en Colombia.

En todo caso, tal como lo mencionan Guerrero y Gómez (2013), el problema de la enseñanza de la ética en la universidad no se reduce a la apertura de nuevos espacios en el currículo ni al diseño estructurado de temarios ni a la intensidad horaria. Esta debe trascender lo meramente descriptivo y enunciativo de la misma y conducir realmente al estudiante a la maduración de estructuras superiores del razonamiento moral y al agenciamiento de las emociones desde las capacidades humanas como modos de florecimiento de la persona. Por eso, como lo anota Nussbaum (2014a), las democracias modernas necesitan de la ética y, en general, de las humanidades, para entenderse a sí mismas y a los otros, a partir de las capacidades humanas y no solo desde las competencias específicas propias de un modelo capitalista.

Finalmente, una cuarta conclusión nos lleva a decir que todos los estamentos ciudadanos coinciden en afirmar que una adecuada formación ética en la universidad constituye un factor importante en la lucha contra la corrupción en Colombia. Sin embargo, esta estrategia pedagógica de modo independiente y aislado no es el antídoto para contrarrestar la corrupción en Colombia; se requiere un trabajo sistemático, estructural y colaborativo que incluya políticas integrales y sistemáticas, leyes más radicales, mayor presencia del Estado en zonas de alto índice de corrupción, cárcel para los corruptos, sanción social a personas y organizaciones que incurran en prácticas corruptas, empoderamiento ciudadano de los recursos públicos, disminución de las brechas salariales para funcionarios públicos, códigos éticos eficaces, políticas de transparencia, rendición de cuentas, mecanismos de seguimiento, etc. Todos estos mecanismos ayudarían a construir lo que Gaviria (2012) denomina fundamentos éticos de la democracia y Nussbaum (2014b), ciudades más compasivas.

En definitiva, ¿qué puede hacer la universidad? El filósofo político de la Universidad del Valle, Delfín Grueso Vanegas, afirma que la universidad tiene la obligación no solo de enseñar ética pública, sino que ella misma debe ser una institución ética y cívica en sus prácticas cotidianas. Esto implica que todos los actores que articulan la universidad (estudiantes, docentes, padres de familia, directivos y entorno social) se unan para transformar las realidades corruptas y apuesten por una sociedad más justa, equitativa y corresponsable donde los principios constitutivos de integridad, transparencia y confianza configuren referentes axiológicos de primer orden. 


\section{Referencias}

Aranguren, José Luis (1998). Ética. Barcelona: Altaya.

Arbeláez-Castaño, Enrique León (2019). Inteligencia emocional. Inédito.

Banco Mundial (1993). World Development Report. Oxford: University Press.

Bauman, Zygmunt; Donskis, Leonidas (2015). Ceguera moral. La pérdida de sensibilidad en la modernidad líquida. Barcelona: Paidós.

Bedoya-Gallego, Nelson; Rey-Vásquez, Elías; Romero-Neisa, William (2015). Las emociones en el desarrollo del juicio moral, en perspectiva de educación filosófica. Análisis, 47(86), 103-118.

Brown, Michael; Mitchell, Marie (2010). Ethical and Unethical Leadership: Exploring New Avenues for Future Research. Business Ethics Quarterly. Revista Electrónica de Investigación Educativa, 20(4), 583-616.

Camps, Victoria (2011). El gobierno de las emociones. Barcelona: Herder.

Camps, Victoria (2013). Breve historia de la ética. Barcelona: RBA Divulgación.

Cepeda-Ulloa, Fernando (2011). Narcotráfico, financiación política y corrupción. Bogotá: ECOE.

Colombia, Corte Constitucional. Sentencia C-113 de 2017. Restricción del derecho de asociación y reunión de menores de edad.

Cortina, Adela (1995). Ética empresarial y opinión pública. Claves de razón práctica, 56, 48-75.

Cortina, Adela (2008). Ética de la empresa. Claves para una nueva cultura empresarial. Madrid: Trotta.

Díaz-Seijas, Raquel (2010). Sobre el gobierno de las emociones de Victoria Camps. Telo. Revista Iberoamericana de Estudios Utilitaristas, 17(2), 229-250.

Escámez, Juan; García, Rafael; Jover, Gonzalo (2008). Restructuring University Degree Programmes: A New Opportunity for Ethics Education? Journal of Moral Education, 37(1), 41-53.

Espinosa-Galán, Víctor Eligio (2015). Formación ética. Educación para la pazy desarrollo moral. Bogotá: Aula de Humanidades.

Euscátegui, Roberto; Pino, Stella; Rojas, Aída (2006). Formación humana en la educación superior. Reflexiones para recrear las estructuras curriculares y repensar las prácticas pedagógicas. Cali: Universidad de San Buenaventura.

Fiscalía General de la Nación (2018). Libro de la indignación. Cien casos de corrupción que han desangrado las arcas del Estado colombiano. Bogotá: Fiscalía General de la Nación. 
Franco-Pérez, Natalia (2017). Atreverse a pensar, un camino hacia la integridad. Medellín: Universidad EAFIT.

García-Villegas, Mauricio; Revelo-Rebolledo, Javier Eduardo (2010). Estado alterado: clientelismo, mafias y debilidad institucional en Colombia. Bogotá: Dejusticia.

Gaviria-Díaz, Carlos (2012). Lectio Inauguralis. Fundamentos éticos de la democracia. Revista de Ciencias Humanas, 8(2), 10-20.

Gilligan, Carol; Attanucci, Jane (1988). Two Moral Orientations - Gender Differences and Similarities. Merrill-Palmer Quarterly of Behavior and Development, 34, 223-237.

Goleman, Daniel (1996). La inteligencia emocional. Barcelona: Kairós.

Guerrero, Manuel (2004). Un estudio psicológico de actitudes corruptas desde la perspectiva de la acción moral. Boletín de Psicología, 8o, 7-36.

Guerrero, Manuel; Galván, Gonzalo; Granados, Alejandro; García, Pablo; Vásquez, Francisco (2018). Validación de un instrumento para medir el desarrollo moral en el contexto de situaciones relacionadas con la corrupción. Psicogente, 21(40), 291-305.

Guerrero-Useda, María Eugenia; Gómez-Paternina, Diomedes Andrés (2013). Enseñanza de la ética y educación moral. ¿Permanecen ausentes de los programas universitarios? Revista Electrónica de Investigación Educativa, 15(1), 122-135.

Habermas, Jürgen (2000). Facticidad y validez sobre el derecho y el Estado democrático de derecho en términos de teoría del discurso. Madrid: Trotta.

Haidt, Jonathan (2001). The Emotional Dog and its Rational Tail: A Social Intuitionist Approach to Moral Judgment. Psychological Review, 108, 814-834.

Hauser, Marc (2006). Moral Minds. How Nature Designed our Universal Sense of Right and Wrong. New York: Harper Collins Publishers.

Henao-Pérez, Juan Carlos (13 de agosto de 2018). Piden menos leyes y más efectividad en la lucha contra corruptos. El Tiempo. Recuperado de https://www.eltiempo.com/justicia/ delitos/jefes-de-organos-de-control-plantean-soluciones-frente-a-la-corrupcion-255330

Henao-Pérez, Juan Carlos; Isaza-Espinoza, Carolina (2018). La corrupción en Colombia (Tomo I). Bogotá: Universidad Externado de Colombia.

Hersh, Richard; Reimert, Joseph; Paolitto, Diana (1998). El crecimiento moral. De Piaget a Kohlberg. Madrid: Narcea.

Honneth, Axel (1997). La lucha por el reconocimiento. Barcelona: Crítica. 
Hoyos-Vásquez, Guillermo; Ruiz-Silva, Alexander (2002). Formación ética, valores y democracia. Recuperado de https://www.oei.es/historico/valores2/catedra_andina/modulo1/ formacion.htm

Ibáñez-Martín, José Antonio (2012). Sentido de la formación ética en la universidad. Revista de Fomento Social, 266, 191-210.

Jiménez, Gonzalo; Sánchez, Omar (19 de agosto de 2018). La consulta. ¿El antídoto seguro? El Colombiano, p. 2.

Keown, John (2004). La eutanasia examinada. Perspectivas éticas, clínicasylegales. México: FCE.

Kohlberg, Lawrence (1969). Stage and Sequence: The Cognitive-Developmental Approach to Socialization. En Handbook of Socialization Theory (pp.347-480), editado por David A. Goslin. Chicago, IL: Rand McNally.

Kohlberg, Lawrence (1992). Psicología del desarrollo moral. Bilbao: Descleé de Brouwer.

Küng, Hans (1997). A Global Ethic in an Age of Globalization. Business Ethics Quaterly, 7(3), 17-32.

Lazarus, Richard (1991). Emotion and Adaptation. New York: Oxford University Press.

Le Breton, David (1998). Las pasiones ordinarias: antropología de las emociones. Buenos Aires: Nueva Visión.

Marreiros, Sofía; Balça, Ángela; Acevedo, Fernando (2010). Confabulando valores: La cigarra y la hormiga. Ocnos: Revista de Estudios sobre Lectura, 6, 61-69.

Martínez, Miquel; Buxarrais, María; Bara, Francisco (2002). La universidad como espacio de aprendizaje ético. Revista Iberoamericana de Educación, 29, 17-43.

Mélich, Joan-Carles (2010). Ética de la compasión. Barcelona: Herder.

Mikhail, John (2007). Universal Moral Grammar: Theory, Evidence and the Future. Trends in Cognitive Sciences, 11(4), 143-152.

Morin, Edgar (2004). Introducción al pensamiento complejo. México: Gedisa.

Newman, Vivian; Ángel, María (2017). Sobre la corrupción en Colombia: marco conceptual, diagnóstico y propuestas de política. Bogotá: La Imprenta.

Nichols, Shaun (2004). Sentimental Rules: On the Natural Foundations of Moral Judgment. Oxford: Oxford University Press.

Nye, Joseph (1967). Corruption and Political Development: A Cost-benefit Analysis. American Political Science Review, 61(2), 417-427. 
Nussbaum, Martha (2005). El cultivo de las humanidades. Una defensa clásica de la reforma en la educación liberal (2. ${ }^{\mathrm{a}}$ ed.). Barcelona: Paidós.

Nussbaum, Martha (2012). Crear capacidades. Propuesta para el desarrollo humano. Barcelona: Paidós.

Nussbaum, Martha (2014a). Sin fines de lucro. Por qué las democracias necesitan de las humanidades. Madrid: Katz.

Nussbaum, Martha (2014b). Emociones politicas. ¿Por qué el amor es importante para la justicia? Bogotá: Planeta.

Nussbaum, Martha (2015). Discurso de Martha Nussbaum al recibir el Doctorado Honoris Causa en Filosofia en la Universidad de Antioquia, el 10 de diciembre de 2015. Recuperado de http:// reservas.parqueexplora.org/visitenos/noticias/discurso-de-martha-nussbaum-al-recibir-el-doctorado-honoris-causa-en-udea/

Organización para la Cooperación y el Desarrollo Económico (OCDE) (2019). Estudio de la OCDE sobre gobernanza pública. La integridad pública en América Latina y el Caribe 20182019. Recuperado de https://www.oecd.org/gov/ethics/integridad-publica-america-latina-caribe-2018-2019.pdf

Orozco-Collazos, Luz Elena (2012). La ética en las empresas. Tendencias en la administración: gerencia y academia. Bogotá: Uniandes.

Orozco-Silva, Luis Enrique (2009). La formación integral. Mito y realidad. Universitas, 10, 161-186.

Ortiz-Gómez, Martha; Velásquez, Jorge Mario (30 de septiembre de 2018). Adela Cortina: la ética da felicidad y es rentable. El Colombiano. Recuperado de http://m.elcolombiano. com/negocios/empresas/adela-cortina-la-etica-da-felicidad-y-es-rentable-XA94062O2

Pascal, Blaise (1959). Pensamientos. El hombre sin Dios y el hombre con Dios (Tomo I). Buenos Aires: Aguilar.

Petrova-Gjeogjeva, Emilija (2010). Democratic Society and Moral Education. Procedia, Social and Behavioral Sciences, 2, 5635-5640.

Piaget, Jean (1965). The Moral Judgement of the Child. New York: Free Press.

Prinz, Jesse (2007). The Emotional Construction of Morals. New York: Oxford University Press.

Rama, Claudio (2004). Ética y educación superior en el contexto de la mercantilización. Universidades, 28, 3-12. Recuperados de http://www.redalyc.org/articulo.oa?id=37302802

Reyes-Solís, Darwin (2017). La educación de las emociones para alcanzar el florecimiento de las capacidades humanas: una mirada desde la filosofia de Martha Nussbaum [Tesis de maestría]. Universidad Pontificia Bolivariana, Medellín. 
Rodríguez-Valls, Francisco (2015). El sujeto emocional. La función de las emociones en la vida humana. Sevilla: Thémata.

Sanagustín, María Victoria (2011). Valores y ética empresarial. Un enfoque sociológico. Madrid: Trotta.

Savater, Fernando (1993). La heterofobia como enfermedad moral. Vuelta, 17(205), 23-27.

Secretaría de Transparencia de la Presidencia de la República (2013). Documento Conpes 167. Recuperado de http://www.anticorrupcion.gov.co/SiteAssets/Paginas/Publicaciones/ Conpes_167.pdf

Solbrekke, Tono; Karseth, Berit (2006). Professional Responsibility - An Issue for Higher Education? Higher Education, 52, 95-119.

Strauss, Anselm; Corbin, Juliet (1998). Basic of Qualitative Research. Techniques and Procedures for Developing Grounded Theory. Thousand Oaks: Sage Publications.

Thoumi, Francisco (1999). La relación entre corrupción y narcotráfico: un análisis general y algunas referencias a Colombia. Revista de Economía de la Universidad del Rosario, 2, 11-33.

Tovar-Bohórquez, José Oliverio (2011). Gramática emocional. Bases cognitivasy sociales del juicio moral [Tesis de doctorado]. Universidad Nacional de Colombia, Bogotá.

Transparency International (2009). Guía de lenguaje claro sobre lucha contra la corrupción. Berlín: Transparency International.

Transparency International (2019). Corruption Perceptions Index 2018. Recuperado de https:// www.transparency.org/cpi2O18

Transparencia por Colombia (2010). Capitulo Transparencia Internacional. Recuperado de http://transparenciacolombia.org.co

Transparencia por Colombia (2019). Índice de Percepción de la Corrupción 2018. Recuperado de http://transparenciacolombia.org.co/2019/02/25/resultados-ipc-2018/

Universidad Pontificia Bolivariana (2012). Formación integral para la transformación socialy humana. Marco conceptual. Medellín: UPB.

Vallaeys, François (2009). El desafío de enseñar ética en la universidad. Papeles de Ética, Economía y Dirección, 4, 1-21.

Vallaeys, François (septiembre, 2018). Ética, responsabilidad e innovación. Trabajo presentado en VI Congreso de Enseñanza de la Ética “Ética de lo público, educación y territorio”, Red para la Formación Ética y Ciudadana, Cali, Colombia. 
Vanegas-Carvajal, Edgar Alonso (2009). La razón y la voluntad en Blaise Pascal. Modos complementarios para el conocimiento. Cali: Universidad de San Buenaventura.

Villegas de Posada, Cristina (2008). La acción moral: explicaciones filosóficas y contrastaciones psicológicas. Bogotá: Uniandes.

Weiss, Joseph (2006). Ética en los negocios: un enfoque de administración de los stakeholdersy de casos. México: Thomson. 